Acta Crystallographica Section D

\title{
Biological
}

Crystallography

ISSN 0907-4449

Editors: E. N. Baker and Z. Dauter

\section{Structural basis for the influence of a single mutation K145N on the oligomerization and photoswitching rate of Dronpa}

\author{
Ngan Nguyen Bich, Benjamien Moeyaert, Kristof Van Hecke, Peter \\ Dedecker, Hideaki Mizuno, Johan Hofkens and Luc Van Meervelt
}

Acta Cryst. (2012). D68, 1653-1659

Copyright (C) International Union of Crystallography

Author(s) of this paper may load this reprint on their own web site or institutional repository provided that this cover page is retained. Republication of this article or its storage in electronic databases other than as specified above is not permitted without prior permission in writing from the IUCr.

For further information see http://journals.iucr.org/services/authorrights.html

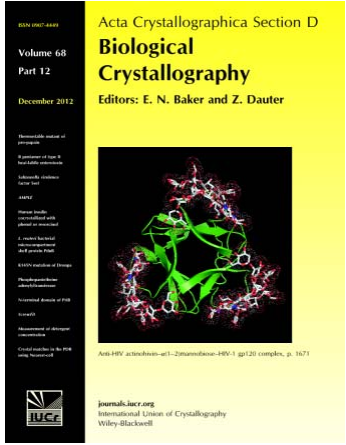

\begin{abstract}
Acta Crystallographica Section D: Biological Crystallography welcomes the submission of papers covering any aspect of structural biology, with a particular emphasis on the structures of biological macromolecules and the methods used to determine them. Reports on new protein structures are particularly encouraged, as are structure-function papers that could include crystallographic binding studies, or structural analysis of mutants or other modified forms of a known protein structure. The key criterion is that such papers should present new insights into biology, chemistry or structure. Papers on crystallographic methods should be oriented towards biological crystallography, and may include new approaches to any aspect of structure determination or analysis. Papers on the crystallization of biological molecules will be accepted providing that these focus on new methods or other features that are of general importance or applicability.
\end{abstract}

Crystallography Journals Online is available from journals.iucr.org 
Acta Crystallographica Section D

Biological

Crystallography

ISSN 0907-4449

Ngan Nguyen Bich, ${ }^{a}$ Benjamien

Moeyaert, ${ }^{\text {b }}$ Kristof Van Hecke, ${ }^{\text {a,c }}$

Peter Dedecker, ${ }^{b}$ Hideaki

Mizuno, Johan Hofkens ${ }^{b}$ and

Luc Van Meervelt ${ }^{a_{*}}$

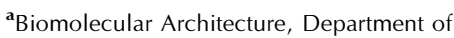
Chemistry, KU Leuven, Celestijnenlaan 200F, B-3001 Leuven (Heverlee), Belgium,

${ }^{\text {b }}$ Photochemistry and Spectroscopy, Department of Chemistry, KU Leuven, Celestijnenlaan 200F,

B-3001 Leuven (Heverlee), Belgium,

${ }^{\mathbf{c}}$ Department of Inorganic and Physical

Chemistry, Ghent University, Krijgslaan 281 S3, B-9000 Gent, Belgium, and ${ }^{\mathbf{d}}$ Biomolecular Network Dynamics, Department of Chemistry, KU Leuven, Celestijnenlaan 200G,

B-3001 Leuven (Heverlee), Belgium

Correspondence e-mail:

luc.vanmeervelt@chem.kuleuven.be

\section{Structural basis for the influence of a single mutation $K 145 \mathrm{~N}$ on the oligomerization and photoswitching rate of Dronpa}

The crystal structure of the on-state of PDM1-4, a singlemutation variant of the photochromic fluorescent protein Dronpa, is reported at $1.95 \AA$ resolution. PDM1-4 is a Dronpa variant that possesses a slower off-switching rate than Dronpa and thus can effectively increase the image resolution in subdiffraction optical microscopy, although the precise molecular basis for this change has not been elucidated. This work shows that the Lys145Asn mutation in PDM1-4 stabilizes the interface available for dimerization, facilitating oligomerization of the protein. No significant changes were observed in the chromophore environment of PDM1-4 compared with Dronpa, and the ensemble absorption and emission properties of PDM1-4 were highly similar to those of Dronpa. It is proposed that the slower off-switching rate in PDM1-4 is caused by a decrease in the potential flexibility of certain $\beta$-strands caused by oligomerization along the $A C$ interface.

\section{Introduction}

Fluorescent proteins (FPs) are a remarkable class of proteins that have revolutionized molecular and cellular imaging. Their typical structure consists of a $\beta$-can structure made up of 11 $\beta$-strands, with an $\alpha$-helix containing the chromophore right at the centre of the structure. This chromophore is formed by the cyclization of a tripeptide with the motif $X Y$ YG. Variations in the identity of the residue $X$ and different conformations and modifications of these chromophores, as well as their interactions with surrounding residues, have resulted in a range of FPs with emission wavelengths spanning almost the entire visible spectrum. Together with the development of imaging techniques, FPs provide a powerful set of tools for tracking the dynamic behaviour of tagged proteins in their cellular environment (see reviews by Wiedenmann et al., 2009; Chudakov et al., 2010; Day \& Davison, 2009). Reversibly photoswitchable FPs (RSFPs), a subclass of FPs, can be changed from a bright fluorescent state to a dim nonfluorescent state and vice versa by irradiation with light of specific wavelengths.

In live cell imaging fluorescent proteins are typically used as fusion tags, which is negatively affected if the fluorescent proteins used have a tendency to oligomerize. Fluorescent proteins derived from the Aquorea victoria GFP, which have little tendency to oligomerize, are easily used as fusion tags. However, most wild-type proteins, especially those cloned from Anthozoa, exist as stable tetramers (Alieva et al., 2008; Nienhaus \& Wiedenmann, 2009) in which each monomer interacts with two adjacent neighbours. It has been shown that mutating certain key residues via rational or random mutagenesis approaches can break these intermolecular interactions, resulting in dimeric or monomeric variants (Wiedenmann et al., 2004; Campbell et al., 2002).
Received 10 July 2012 Accepted 18 September 2012

PDB Reference: PDM1-4, 4emq
(C) 2012 International Union of Crystallography Printed in Singapore - all rights reserved 
Table 1

Data-collection and refinement statistics for PDM1-4.

Values in parentheses are for the highest resolution shell.

\begin{tabular}{|c|c|}
\hline PDB entry & $4 \mathrm{emq}$ \\
\hline Space group & $P 22_{1} 2_{1}$ \\
\hline Unit-cell parameters $\left(\AA,^{\circ}\right)$ & $\begin{array}{c}a=69.54, b=103.80, c=177.25 \\
\quad \alpha=\beta=\gamma=90.0\end{array}$ \\
\hline Resolution range $(\AA)$ & $54.93-1.95(2.06-1.95)$ \\
\hline$R_{\text {merge }}(\%)$ & $11.5(86.4)$ \\
\hline$R_{\text {p.i.m. }}(\%)$ & $4.5(34.0)$ \\
\hline$\langle I / \sigma(I)\rangle$ & $15.0(2.5)$ \\
\hline No. of unique reflections & 94305 (13605) \\
\hline Multiplicity & $7.4(7.4)$ \\
\hline Completeness (\%) & $100.0(100.0)$ \\
\hline \multicolumn{2}{|l|}{ Refinement statistics } \\
\hline Resolution $(\AA)$ & $54.71-1.95$ \\
\hline$R_{\text {work }} / R_{\text {free }} \dagger(\%)$ & $18.35 / 23.24$ \\
\hline \multicolumn{2}{|l|}{ R.m.s.d. from ideal geometry } \\
\hline Bond lengths $(\AA)$ & 0.009 \\
\hline Bond angles $\left({ }^{\circ}\right)$ & 1.30 \\
\hline No. of protein atoms & 10403 \\
\hline No. of waters & 884 \\
\hline No. of ligands & 125 \\
\hline \multicolumn{2}{|l|}{ Average $B$ factors $\left(\AA^{2}\right)$} \\
\hline Main chain & 27.3 \\
\hline Side chain & 29.5 \\
\hline Waters & 32.6 \\
\hline Ligands & 44.0 \\
\hline \multicolumn{2}{|l|}{ Ramachandran plot $¥(\%)$} \\
\hline Residues in favoured regions & 98.9 \\
\hline Residues in allowed regions & 1.1 \\
\hline Outliers & 0.0 \\
\hline
\end{tabular}

$\dagger R_{\text {free }}$ is $R$ calculated using a random $5 \%$ of data that were excluded from refinement. $\$$ Ramachandran analysis was carried out using MolProbity (Chen et al., 2010).

In the course of this progress, Dronpa, a monomeric efficient RSFP, was engineered from a tetrameric FP cloned from Pectiniidae sp., 22G (Ando et al., 2004). Apart from being brightly fluorescent, Dronpa has the remarkable property of being reversibly photoswitchable. While exciting the absorption band with a maximum at $503 \mathrm{~nm}$ results in strong green fluorescence peaking at $518 \mathrm{~nm}$, strong illumination can turn Dronpa into a dark, non-emissive state characterized by an absorption band centred at $390 \mathrm{~nm}$. Irradiating this band with only low light intensity quickly converts the dark state back to the bright fluorescent state. As the photoswitching behaviour is fully reversible, this cycle can be repeated many times (Ando et al., 2004). The chromophore of Dronpa, i.e. a \{(4Z)2-[1-amino-2-mercaptoethyl]-4-(4-hydroxybenzylidene)-5-oxo4,5-dihydro- $1 H$-imidazol-1-yl\}-acetaldehyde moiety, is formed spontaneously from the tripeptide Cys62-Tyr63-Gly64, referred to as GYC; it is part of an $\alpha$-helix and is positioned in the centre of the $\beta$-can. It has been demonstrated that cistrans isomerization and protonation and deprotonation of this bicyclic chromophore is a key event in the switching process of Dronpa (Habuchi et al., 2005; Stiel et al., 2007; Wilmann et al., 2006; Andresen et al., 2007). Additionally, certain residues in its vicinity have been shown to have a crucial influence on its photoswitchable properties (Wilmann et al., 2006).

Recently, NMR studies conducted by Mizuno and coworkers (Mizuno et al., 2008; Mizuno, Mal et al., 2010) have demonstrated that the photochromic mechanism of Dronpa is intimately linked to changes in the flexibility of the surrounding structure involving part of the $\beta$-barrel. Notably, Mizuno, Dedecker et al. (2010) demonstrated that the oligomerization status of an RSFP could influence its off-switching rate. By comparing the photochromic properties of $22 \mathrm{G}$ and of its monomeric variant Dronpa, it was proposed that the tetrameric form is almost nonphotoswitchable, while the monomeric form displays much faster switching kinetics. In the same work, PDM1-4 was presented. This point mutant of Dronpa (Lys145Asn) was an RSFP created by random mutagenesis and was selected to have an off-switching rate that is intermediate between those of $22 \mathrm{G}$ and Dronpa. While its precursor Dronpa is almost exclusively present as a monomer in solution, PDM1-4 shows a tendency to oligomerize into a tetrameric form. The mutation is located on the exterior of the $\beta$-barrel rather than in the microenvironment of the chromophore. This raises the question of how this mutation can influence the off-switching rate and whether the self-association plays any role in this relationship.

Here, we present the $1.95 \AA$ resolution structure of PDM1-4 in the green-fluorescent state and address the role of the single mutation in the self-association of PDM1-4. Moreover, we show that the chromophore environment as well as the spectroscopic parameters are practically identical, which proves our point of view that the photoswitching kinetics are related to the flexibility of part of the $\beta$-barrel.

\section{Materials and methods}

\subsection{Protein production}

PDM1-4 was previously created by error-prone PCR and characterized by a screening setup (Mizuno, Dedecker et al., 2010). For X-ray crystallography, the protein was expressed in Escherichia coli strain JM109 (DE3) (Promega, Madison, Wisconsin, USA) and a single colony was inoculated into $300 \mathrm{ml}$ Luria-Bertani medium supplemented with ampicillin and incubated at $294 \mathrm{~K}$ for $72 \mathrm{~h}$ with strong shaking. Cells were then harvested and disrupted using repeated freezethaw cycles in the presence of lysozyme.

Purification by affinity chromatography was performed using HisTrap FF crude columns on an ÄKTAprime system. Fractions that were visibly green were pooled and were further purified using a Superdex 200 size-exclusion chromatography column on an ÄKTApurifier system (all from GE Healthcare) using a buffer consisting of $30 \mathrm{~m} M \mathrm{NaCl}, 10 \mathrm{~m} M$ Tris pH 7.4. The resulting solution was concentrated to $4 \mathrm{mg} \mathrm{ml}^{-1}$ using a Vivaspin membrane ultrafiltration tube (10 000 molecularweight cutoff; Sartorius Stedim). The protein concentration was calculated from the absorption spectrum (Shimadzu UV1650 PC) using the extinction coefficient reported here.

\subsection{Crystallization}

Crystallization conditions for PDM1-4 were screened by the hanging-drop vapour-diffusion method using the 96-condition Index screen (Hampton Research, Aliso Viejo, USA). Hanging drops consisting of $1 \mu \mathrm{l}$ PDM1-4 $\left(4 \mathrm{mg} \mathrm{ml}^{-1}\right)$ in $30 \mathrm{~m} M \mathrm{NaCl}, 10 \mathrm{~m} M$ Tris $\mathrm{pH} 7.4$ and $1 \mu$ well solution were 
equilibrated against $500 \mu \mathrm{l}$ screen solution in 24-well ComboPlates (Greiner Bio-One, Wemmel, Belgium) at $289 \mathrm{~K}$. Green-fluorescent PDM1-4 crystals suitable for X-ray diffraction were obtained after approximately two weeks in the condition $25 \%(w / v)$ PEG 3350, 0.1 $M$ HEPES pH 7.5. The crystals were swept through cryoprotectant $[12.5 \%(w / v)$ PEG 3350, $0.05 M$ HEPES pH 7.5, 20\%(v/v) glycerol] and flashcooled in liquid nitrogen prior to X-ray data collection.

\subsection{Data collection and structure determination}

2.3.1. Data collection. Data were collected on a MAR 225 CCD detector using a wavelength of $1.00 \AA$, a $\varphi$ range of $180^{\circ}$, an increment of $0.75^{\circ}$ and a crystal-to-detector distance of $180 \mathrm{~mm}$ in a liquid-nitrogen cryostream at $100 \mathrm{~K}$ on beamline PXIII of the SLS synchrotron, Villigen, Switzerland. Data were processed with $X D S$ v.December 62010 (Kabsch, 2010) and scaled with $S C A L A$ v.3.3.16 (Evans, 2006). Although the resolution edge of the diffraction pattern was $1.80 \AA$, the data were truncated to $1.95 \AA$ resolution as the $R_{\text {merge }}$ increased significantly at higher resolution. The crystal belonged to the orthorhombic space group $P 22_{1} 2_{1}$, with unit-cell parameters $a=69.54, b=103.80, c=177.25 \AA$. The data-collection statistics are summarized in Table 1.

2.3.2. Structure determination. The structure of PDM1-4 was solved by the molecular-replacement method using the program Phaser v.2.1.4 (McCoy et al., 2007). The coordinates of chain $A$ of Dronpa (PDB entry 2z1o; Mizuno et al., 2008) were used as the search model with the chromophore GYC omitted. Both the chromophore and the mutated residue were modelled into the unambiguous electron-density map with the program Coot v.0.6.1 (Emsley et al., 2010) after subsequent refinement loops. Each loop included three macrocycles of bulk-solvent and anisotropic scaling, individual coordinate and isotropic $B$-factor refinements and refinement of occupancies for atoms in alternative conformations performed using the phenix.refine GUI (Afonine et al., 2005). The standard dictionary files were used, with an additional dictionary entry for the GYC chromophore moiety and ligands, including di(hydroxyethyl)ether (PEG), triethylene glycol (PGE), tetraethylene glycol (PG4) and HEPES (EPE), created by eLBOW (Moriarty et al., 2009). Average target values for the imidazolinone and hydroxyphenyl rings of the chromophore were obtained from the Cambridge Structural Database (Allen, 2002).

The likelihood-based refinement converged to $R_{\text {work }}$ and $R_{\text {free }}$ values of $18.35 \%$ and $23.24 \%$, respectively. Water molecules were included in the model if they were within hydrogen-bonding distance of chemically reasonable groups, appeared in $m F_{\mathrm{o}}-D F_{\mathrm{c}}$ maps contoured at $3.0 \sigma$ and had a $B$ factor of less than $80 \AA^{2}$ using the water-picking mode in the phenix.refine GUI. A total of six fragments of polyethylene glycol, including PEG, PGE and PG4, were found and placed reasonably in the electron-density maps during the progress of refinement. Six HEPES molecules were also found and modelled in the large blobs consistent with the $\mathrm{S}$ atoms of the sulfate head of HEPES; the tails showed considerable disorder. Refinement statistics are given in Table 1.

\subsection{Spectroscopic characterization}

Steady-state absorption spectra of Dronpa and PDM1-4 were obtained using a Shimadzu UV-1650 PC spectrophotometer and a PTI QuantaMaster fluorimeter. The extinction

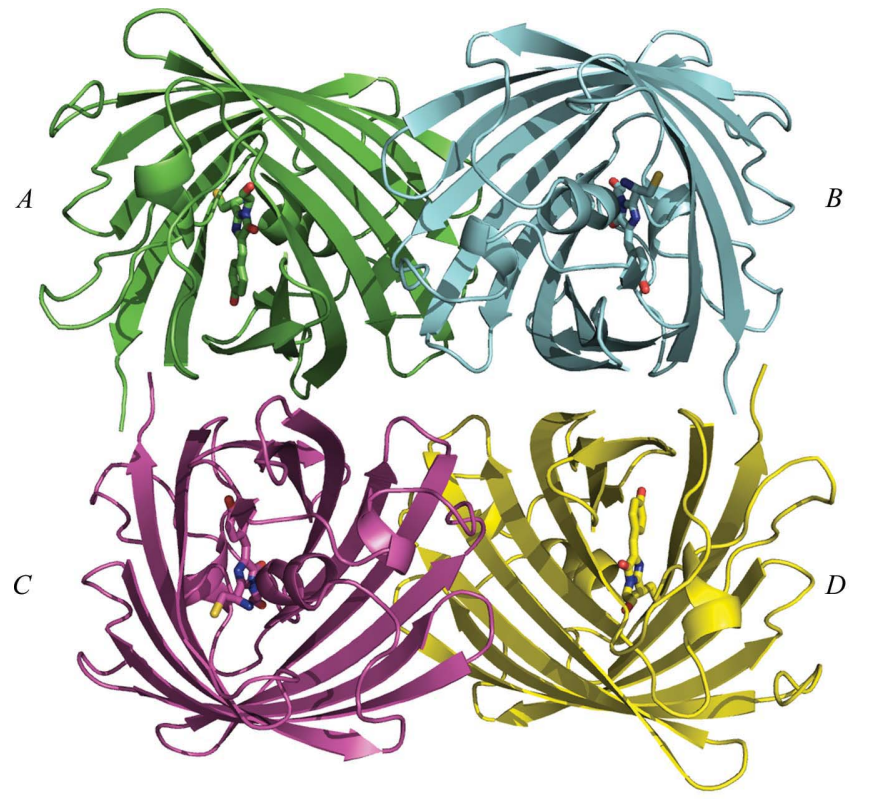

(a)

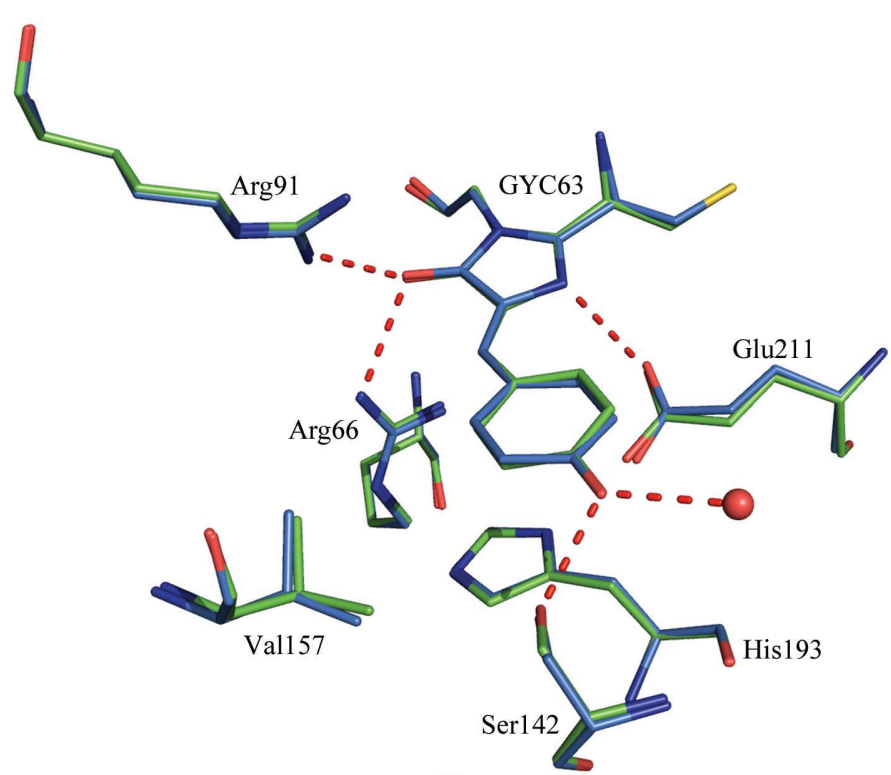

(b)

Figure 1

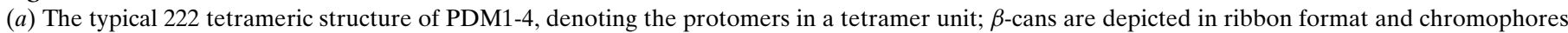

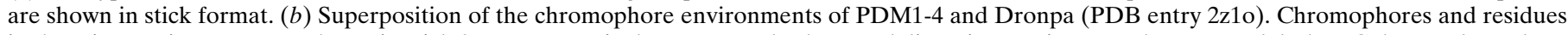

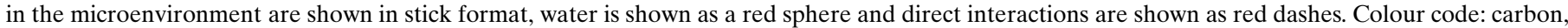
green (PDM1-4) and light blue (Dronpa); nitrogen, dark blue; oxygen, red; sulfur, yellow. 
coefficient of PDM1-4 was determined using the method of Ward et al. (1980) using the literature value for Dronpa (95000 $\mathrm{M}^{-1} \mathrm{~cm}^{-1}$ ) as a reference. The quantum yield of fluorescence (QY) of both proteins was determined relative to that of fluorescein in $0.1 \mathrm{M} \mathrm{NaOH}$ (QY of 0.925 ). The excitedstate lifetime was measured and fitted as described previously (Adam et al., 2011).

\section{Results and discussion}

\subsection{Overall structure of PDM1-4}

Each protomer has an 11 -stranded ' $\beta$-can' fold similar to that described for Dronpa and GFP-like proteins, together with interconnecting loops and a small $\alpha$-helix. The chromophore is found at the centre of the $\beta$-barrel. Six protomers are found in an asymmetric unit and form one and a half tetramers; the second half of the tetramer is generated by a crystallographic twofold axis. Although the six protomers show noncrystallographic symmetry (NCS), they were refined separately without applying NCS restraints during refinement. Their structures are almost identical (pairwise r.m.s.d. of 0.16$0.35 \AA$ for 213-214 matching $\mathrm{C}^{\alpha}$ atoms).

Previously reported analytical ultracentrifugation experiments showed that the tetrameric form of PDM1-4 is

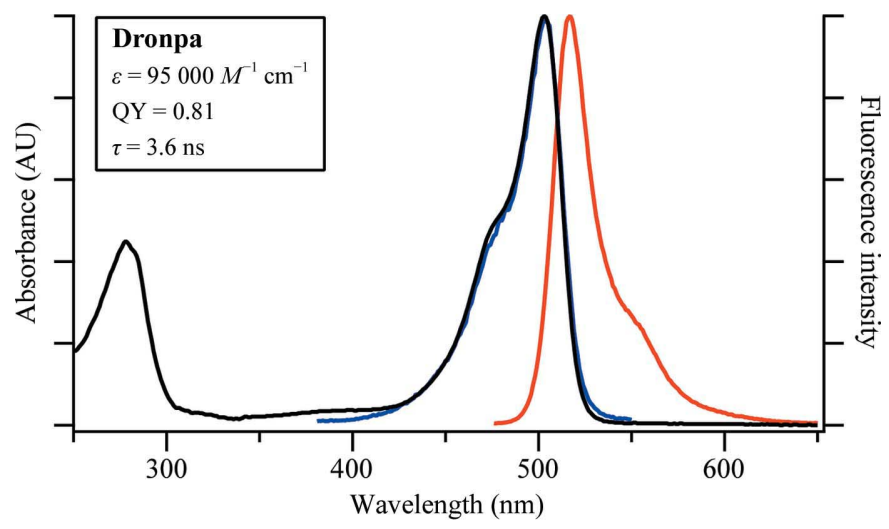

(a)

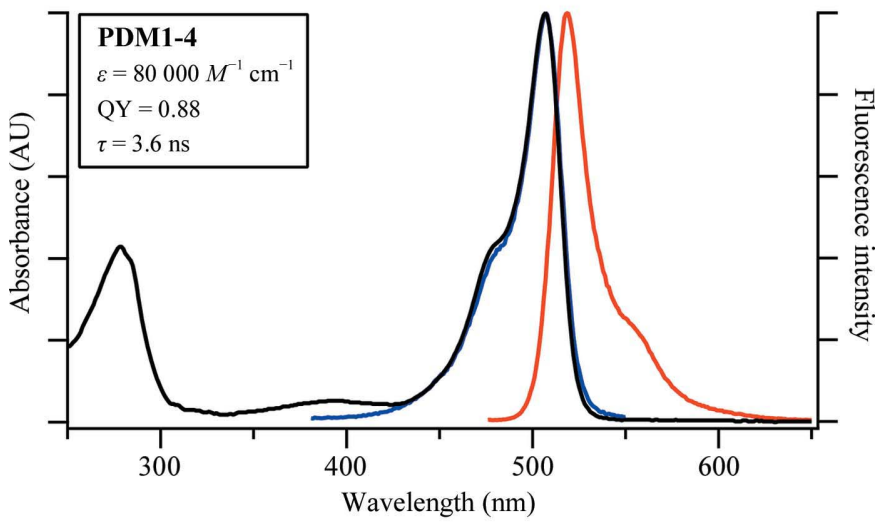

(b)

Figure 2

Spectra of the on-states of (a) Dronpa and (b) PDM1-4 (black lines, absorbance; blue lines, excitation; red lines, emission spectra). $\varepsilon$, extinction coefficient; QY, quantum yield of fluorescence; $\tau$, excitedstate lifetime. dominant at concentrations of $0.1 \mathrm{~m} M$ or greater (Mizuno, Dedecker et al., 2010). In the crystal lattice, PDM1-4 forms a typical $D_{2}$-symmetry quaternary structure representative of the tetrameric form in solution (Fig. 1a). The orientations of the four protomers in a unit of oligomerized-state PDM1-4 are considered to be identical to those of Dronpa and its ancestor 22G (Mizuno et al., 2008; r.m.s.d.s of 0.38 and $0.47 \AA$, respectively, for $853 \mathrm{C}^{\alpha}$ atoms).

\subsection{Chromophore structure and environment}

After the first round of cycles of refinement of the model lacking the chromophores, they could be unambiguously modelled in the $m F_{\mathrm{o}}-D F_{\mathrm{c}}$ map in all six copies of the protein. The chromophore GYC is part of an $\alpha$-helix and is positioned in the centre of the $\beta$-can.

The hydrogen-bonding network among the chromophore, the surrounding residues and certain water molecules is conserved from its ancestors and stabilizes the chromophore inside the $\beta$-barrel, thus allowing the chromophore to fluoresce (Fig. 1b). As in Dronpa, the side chain of Ser142 forms a hydrogen bond to the hydroxyl group of the $p$-hydroxyphenyl ring of the chromophore. This is accompanied by watermediated interactions with the backbone carbonyl group of Glu140 (in $\beta 7$ ) and the amino group of Ile195 (in $\beta 10$ ), maintaining the stabilization of its cis conformation. This sixmembered ring is sandwiched by the imidazole ring of His193 and the aliphatic moiety of Thr159. The chromophore imidazolinone ring is maintained in a hydrogen-bonding network by direct interactions with the side chains of Arg66, Arg91 and Glu211, with the backbone carbonyl group of Thr59 and by water-mediated contacts with the side chains of Gln38, Arg66 and Glu211 and the backbone carbonyl group of Asn65.

The chromophore environments of Dronpa and PDM1-4 are strikingly similar (Fig. 1b). Steady-state and nanosecond time-resolved spectroscopy showed that not only are the absorption and emission spectra almost identical, but that the quantum yield, extinction coefficient and excited-state lifetime, which are properties that are highly dependent on the chromophore environment, are also practically identical (Fig. 2).

\subsection{Influence of the mutation on oligomerization}

The typical quaternary structure of GFP-like proteins from Anthozoa consists of four protomers/chains stabilized by pairs of $A C(B D)$ and $A B(C D)$ interfaces. This was first reported in the structure of DsRed (Wall et al., 2000). The PDM1-4 AB interface is formed around the pairwise interaction of Asn102/ Asn102, Asp121/Asp121 and Arg119/Arg119, together with hydrophobic interactions among the side chains of residues in the $\beta 4-\beta 6$ strands (cyan residues in Figs. $3 a$ and $3 b$ ). The $A C$ interface is formed by hydrophilic interactions between the $\beta 7-\beta 10$ strands of the two neighbouring protomers with the centre region around the twofold axis, including the symmetric Thr143/Thr143, Asn158/Asn158 and Asp172/Asp172 pairs (Figs. $3 c$ and $3 d$ ). Moreover, the interface is stabilized by both electrostatic interactions and a number of hydrogen bonds 
among the polar uncharged/charged residues (green, blue and red residues in Figs. $3 c$ and $3 d$ ).

In most reports regarding the monomerization of an originally tetrameric fluorescent protein, key apolar or uncharged polar residues in the interfaces are mutated to polar or charged polar residues, respectively, to obtain more hydrophilic interfaces (Day \& Davison, 2009; Campbell et al., 2002; Wiedenmann et al., 2004; Habuchi et al., 2008). This was applied in the monomerization of $22 \mathrm{G}$ to create the monomeric variant Dronpa. The $A B$ interface of $22 \mathrm{G}$ was disrupted by the mutation Ile102Asn, while the $A C$ interface was disrupted by the mutation Gly218Glu, which broke the 'armin-arm' configuration (Ando et al., 2004; Mizuno et al., 2008). Indeed, the oligomerization of fluorescent proteins can be stimulated by applying the reverse of these mutations, which means introducing fewer hydrophilic interfaces or removing degrees of freedom of specific side chains.

The outward-facing region of PDM1-4 and Dronpa, which belongs to the $A C$ interface in the monomeric state in solution, is dominated by charged residues, including Glu140,
Lys145 in Dronpa/Asn145 in PDM1-4, Asp156, His168, Arg170 and Asp172. At this $A C$ interface, Glu140 and Asp172 are fixed by interaction with His168 and Arg170, respectively, through an electrostatic interaction, while Asp156 forms a hydrogen bond to Tyr147. These interactions on one hand limit the side-chain degrees of freedom of these charged residues and on the other hand neutralize the electric charge of the surface, making it less hydrophilic. Lys145, however, is not tied by any interaction. Its side chain has a high degree of freedom compared with the others, which contributes to the repulsion of one protomer by another. When replacing Lys145 by Asn145, which is modelled unambiguously in the OMIT map (Fig. 4a), we reason that these effects are largely eliminated. On one hand, the polar uncharged residue no longer contributes to the total charge of this outer face and, on the other hand, the shorter side chain of Asn has fewer degrees of freedom compared with the longer side chain of Lys145 in Dronpa. Moreover, the Asn145 (in $\beta 7$ ) side chain is fixed tightly by the side chain of Tyr188 (in $\beta 10$ ) and the backbone carbonyl group of Asp156 (in $\beta 8$ ) of the same chain (Fig. 4b).

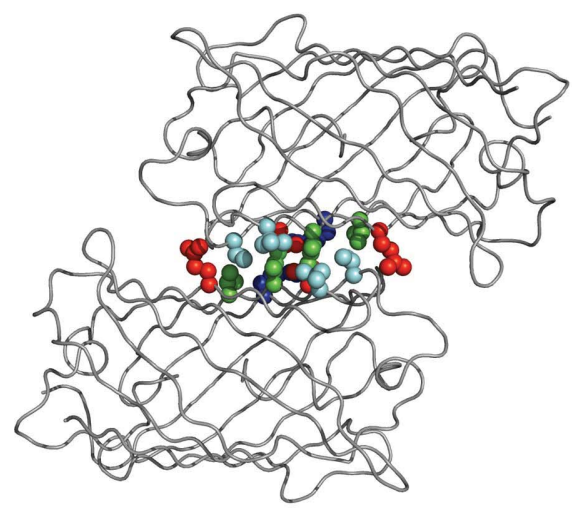

(a)

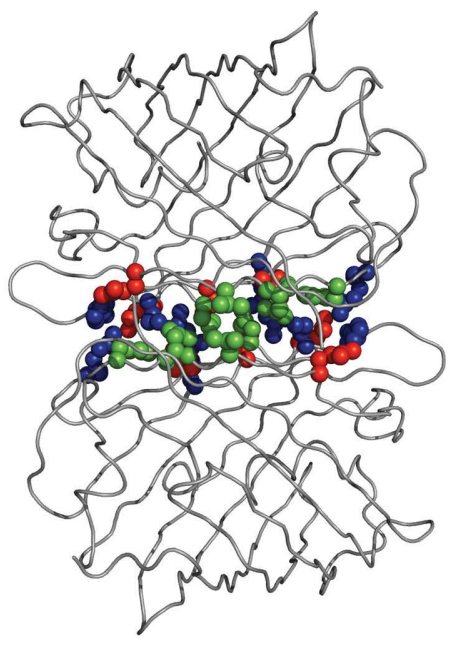

(c)

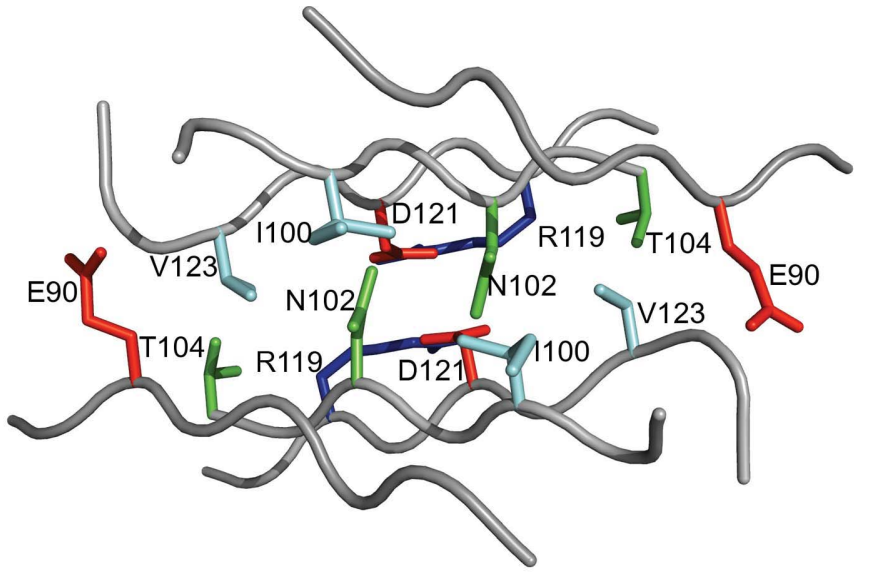

(b)

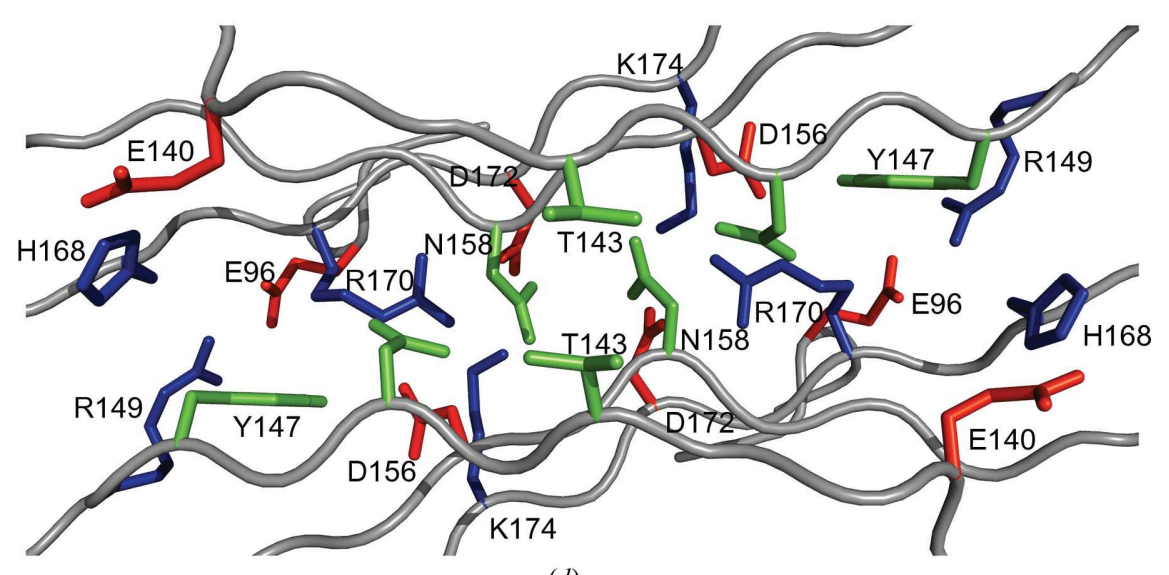

(d)

Figure 3

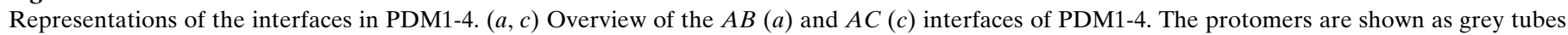

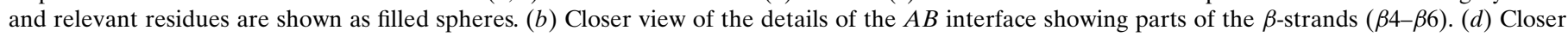

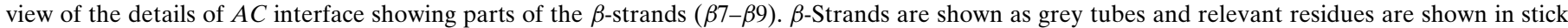
representation. Colour code: apolar residues, cyan; polar uncharged residues, green; negative charged residues, red; positive charged residues, blue. 
A more rigid residue at position 145 of PDM1-4 compared with Dronpa is favourable for increasing the capacity of the protomers to create oligomers through the $A C$ interface in solution. Additionally, the interaction of the Asn145 side chain with the side chain of Asn158 of the adjacent protomer (Fig. $4 b$ ) is favourable for stabilizing the $A C$ interface.

Although position 145 is not a central residue of the $A C$ interface, it is proximate to the central residues and has an influence on the self-association of PDM1-4. Mizuno, Dedecker et al. (2010) showed that Dronpa is in monomeric and dimeric forms in solution depending on concentration, while PDM1-4 is mostly monomeric and tetrameric. We suppose that the $A B(C D)$ dimers are formed dominantly in Dronpa at high concentration; this was also observed in the crystal lattice, as shown in PDB entries 2iov (Stiel et al., 2007) and 2pox (Andresen et al., 2007). In PDM1-4, however, Asn145 is favourable for formation of the $A C(B D)$ interface and this explains why dimers are not observed at higher concentrations, as they immediately associate to form tetramers.

\subsection{The influence of oligomerization on the photoswitching kinetics in relation to the flexibility of the $\beta$-barrel}

PDM1-4 qualitatively exhibits the same photochromism as Dronpa (Fig. 2), except for a slower off-switching rate, which was considered to derive from its capability to self-associate (Mizuno, Dedecker et al., 2010). Moreover, superimposition of the chromophore structure and its microenvironment in Dronpa and PDM1-4 shows that these are almost identical (Fig. 1b). This is consistent with the point of view that the switching kinetics are not only governed by the nature of the chromophore and its microenvironment, but also by the flexibility of a certain part of the $\beta$-barrel resulting from the structural decoupling of the chromophore and this specific part of the $\beta$-barrel, as described by Mizuno and coworkers (Mizuno et al., 2008; Mizuno, Mal et al., 2010). Remarkable flexibility of the $\beta$-barrel was observed in the off-state of Dronpa, mostly located in the $\beta 7-\beta 11$ strands (Figs. $2 a, 2 b$ and $2 c$ in Mizuno et al., 2008).

Careful inspection of the PDM1-4 and Dronpa structures shows that the dimerization of Dronpa along the $A B(C D)$ interface probably does not influence the switching kinetics, as the $A B(C D)$ interface involves three $\beta$-strands, $\beta 4-\beta 6$, that hardly interact with the chromophore. This is also consistent with the fact that the off-switching properties of Dronpa are not influenced by the formation of $A B$ dimers. However, in PDM1-4 the Lys145Asn mutation directly stimulates the formation of tetramers, creating a more rigid organization along the $\beta 7-\beta 9$ strands of the $A C(B D)$ interface and directly resulting in a slower off-switching rate.

Asn145 is also involved in the formation of a tight network that keeps the crucial $\beta$-strands in a rather rigid conformation. It is positioned in $\beta 7$, the same $\beta$-strand as Ser142, the side chain of which tightly keeps the chromophore in the fluorescent cis-conformation (Fig. 1b). Moreover, Asn145 interacts directly with Asn156 located in $\beta 8$ (Fig. 4b), which neighbours Val157, the main steric counteraction to the cis-trans isomerization of the chromophore, as reported previously by Andresen et al. (2007) and Stiel et al. (2007). They demonstrated that Ser142 and Val157 were the most movable residues (0.5 and $1.0 \AA$ shifts for $\mathrm{C}^{\alpha}$, respectively) for enlarging the chromophore cavity when switching from cis on-state to the trans off-state of Dronpa. All of these residues are located in the $\beta$-strands, becoming flexible when switching to the offstate of Dronpa. Consequently, the more rigid conformation of PDM1-4 in $\beta 7-\beta 9$ (average $B$ factors of $22.4 \AA^{2}$ for $\beta 7-\beta 9$ and $24.7 \AA^{2}$ for all $\beta$-strands) together with the formation of the $A C$ interface could be unfavourable for the switch from the on-state to the flexible off-state, as indicated by the slower off-switching rate of PDM1-4. The effect of Asn145 contributes to the rigidity of all $\beta$-strands in the $A C$ interface, not only to $\beta 7$. This could be the reason why we did not observe a significant decrease in the relative crystallographic

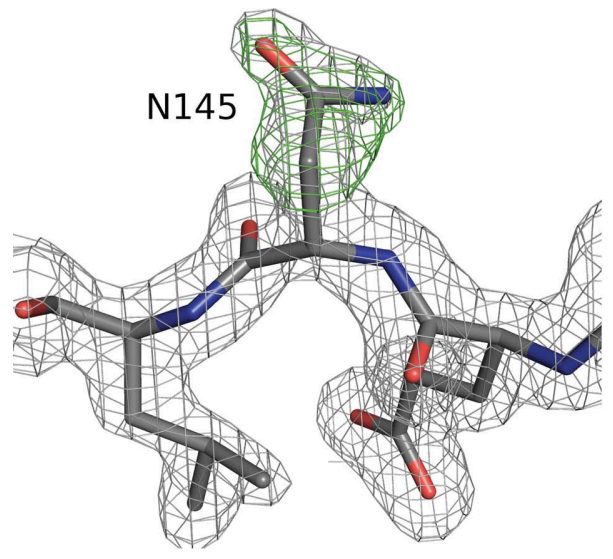

(a)

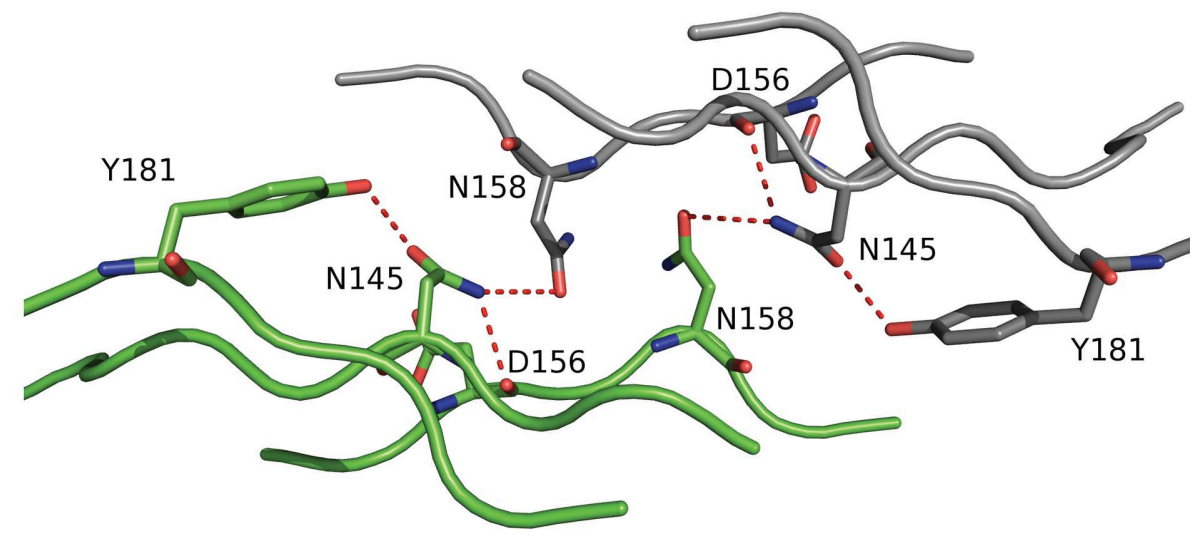

(b)

Figure 4

(a) View of Asn145 with superposed $2 m F_{\mathrm{o}}-D F_{\mathrm{c}}$ electron-density map (grey mesh) and OMIT map $\left(m F_{\mathrm{o}}-D F_{\mathrm{c}}\right.$; green mesh) contoured at $1.5 \sigma$ and $5.0 \sigma$, respectively. $(b)$ View of Asn145 interactions within the $A C$ interface showing parts of the $A$ protomer (green tube) and the $C$ protomer (grey tube) and the pairs of residues (sticks) Asn158/Asn158, Tyr188/Tyr188 and Asp156/Asp156 making interactions (red dashes) with Asn145/Asn145. 
temperature factors ( $B$ factors) of the $\beta$-strands in the $A C$ interface of PDM1-4 compared with those of Dronpa, as was the case in the structure of mTurquoise2 (Goedhart et al., 2011).

These findings give us a new insight into the switching mechanism of RSFPs. Previously, the switching kinetics were thought to be mainly influenced by the interaction of the chromophore with its microenvironment. For instance, DronpaM159T (Dronpa-2), DronpaV157I/M159A (Dronpa-3; Ando et al., 2007) and DronpaV157G (rsFastLime; Stiel et al., 2007) have been shown to have faster off-switching rates compared with Dronpa, which was attributed to lower steric repulsion on isomerization of the chromophore. However, our crystal structure and spectroscopic data show that the slower off-switching of PDM1-4 can be attributed to a lower possibility of switching to the more flexible off-state by means of oligomer formation. Indeed, the Lys145Asn mutation does not significantly alter the chromophore environment, neither does it appreciably influence the ensemble spectroscopic properties of Dronpa. The sole effect of the Lys145Asn mutation located at the exterior of the $\beta$-barrel is thus to directly cause oligomerization of PDM1-4, indirectly affecting the off-switching rate by modulating the rigidity of $\beta$-strands in the $A C(B D)$ interface.

\section{Conclusions}

We have presented the X-ray diffraction structure of the reversibly photoswitchable fluorescent protein PDM1-4. The single mutation Lys145Asn on the exterior of the $\beta$-can of Dronpa makes PDM1-4 tend to oligomerize into a tetrameric form, as has been shown in the present structure. While the structure of the chromophore and its environment, as well as several critical spectroscopic parameters, do not significantly differ compared with Dronpa, the switching kinetics of PDM1-4 are markedly different. Our results support the point of view that the flexibility of the $\beta$-strands at the $A C$ interface influences the switching kinetics of reversibly photoswitchable fluorescent proteins. These findings suggest that any mutation that affects the flexibility of the $\beta$-barrel, either directly or indirectly by influencing the oligomerization behaviour, could result in a change in the photoswitching kinetics of reversibly photoswitchable fluorescent proteins.

The authors thank the staff of the Swiss Light Source in Villigen (beamline PXIII) for help with the synchrotron experiments. We also thank Dr A. Miyawaki for providing us with the plasmid PDM1-4/pRSET. This work was partly supported by grants from the Vietnamese government and the Katholieke Universiteit Leuven. PD is a postdoctoral fellow of the Research Foundation-Flanders (FWO Vlaanderen). BM is funded by a $\mathrm{PhD}$ grant from the Agency for Innovation by Science and Technology (IWT) Flanders.

\section{References}

Adam, V., Moeyaert, B., David, C. C., Mizuno, H., Lelimousin, M., Dedecker, P., Ando, R., Miyawaki, A., Michiels, J., Engelborghs, Y. \& Hofkens, J. (2011). Chem. Biol. 18, 1241-1251.

Afonine, P. V., Grosse-Kunstleve, R. W. \& Adams, P. D. (2005). CCP4 Newsl., 42, contribution 8.

Alieva, N. O., Konzen, K. A., Field, S. F., Meleshkevitch, E. A., Hunt, M. E., Beltran-Ramirez, V., Miller, D. J., Wiedenmann, J., Salih, A. \& Matz, M. V. (2008). PLoS One, 3, e2680.

Allen, F. H. (2002). Acta Cryst. B58, 380-388.

Ando, R., Flors, C., Mizuno, H., Hofkens, J. \& Miyawaki, A. (2007). Biophys. J. 92, 97-99.

Ando, R., Mizuno, H. \& Miyawaki, A. (2004). Science, 306, 13701373.

Andresen, M., Stiel, A. C., Trowitzsch, S., Weber, G., Eggeling, C., Wahl, M. C., Hell, S. W. \& Jakobs, S. (2007). Proc. Natl Acad. Sci. USA, 104, 13005-13009.

Campbell, R. E., Tour, O., Palmer, A. E., Steinbach, P. A., Baird, G. S., Zacharias, D. A. \& Tsien, R. Y. (2002). Proc. Natl Acad. Sci. USA, 99, 7877-7882.

Chen, V. B., Arendall, W. B., Headd, J. J., Keedy, D. A., Immormino, R. M., Kapral, G. J., Murray, L. W., Richardson, J. S. \& Richardson, D. C. (2010). Acta Cryst. D66, 12-21.

Chudakov, D. M., Matz, M. V., Lukyanov, S. \& Lukyanov, K. A. (2010). Physiol. Rev. 90, 1103-1163.

Day, R. N. \& Davison, M. W. (2009). Chem. Soc. Rev. 38, 2887-2921.

Emsley, P., Lohkamp, B., Scott, W. G. \& Cowtan, K. (2010). Acta Cryst. D66, 486-501.

Evans, P. (2006). Acta Cryst. D62, 72-82.

Goedhart, J., von Stetten, D., Noirclerc-Savoye, M., Lelimousin, M., Joosen, L., Hink, M. A., von Weeren, L., Gadella, T. W. J. Jr \& Royant, A. (2011). Nature Commun. 3, 751.

Habuchi, S., Ando, R., Dedecker, P., Verheijen, W., Mizuno, H., Miyawaki, A. \& Hofkens, J. (2005). Proc. Natl Acad. Sci. USA, 102, 9511-9516.

Habuchi, S., Tsutsui, H., Kochaniak, A. B., Miyawaki, A. \& van Oijen, A. M. (2008). PLoS One, 3, e3944.

Kabsch, W. (2010). Acta Cryst. D66, 125-132.

McCoy, A. J., Grosse-Kunstleve, R. W., Adams, P. D., Winn, M. D., Storoni, L. C. \& Read, R. J. (2007). J. Appl. Cryst. 40, 658-674.

Mizuno, H., Dedecker, P., Ando, R., Fukano, T., Hofkens, J. \& Miyawaki, A. (2010). Photochem. Photobiol. Sci. 9, 239-248.

Mizuno, H., Mal, T. K., Wälchli, M., Fukano, T., Ikura, M. \& Miyawaki, A. (2010). J. Biomol. NMR, 48, 237-246.

Mizuno, H., Mal, T. K., Wälchli, M., Kikuchi, A., Fukano, T., Ando, R., Jeyakanthan, J., Taka, J., Shiro, Y., Ikura, M. \& Miyawaki, A. (2008). Proc. Natl Acad. Sci. USA, 105, 9227-9232.

Moriarty, N. W., Grosse-Kunstleve, R. W. \& Adams, P. D. (2009). Acta Cryst. D65, 1074-1080.

Nienhaus, G. U. \& Wiedenmann, J. (2009). ChemPhysChem, 10, 1369 1379.

Stiel, A. C., Trowitzsch, S., Weber, G., Andresen, M., Eggeling, C., Hell, S. W., Jakobs, S. \& Wahl, M. C. (2007). Biochem. J. 402, 35-42.

Wall, M. A., Socolich, M. \& Ranganathan, R. (2000). Nature Struct. Mol. Biol. 7, 1133-1138.

Ward, W. W., Cody, C. W., Hart, R. C. \& Cormier, M. J. (1980). Photochem. Photobiol. 31, 611-615.

Wiedenmann, J., Ivanchenko, S., Oswald, F., Schmitt, F., Röcker, C., Salih, A., Spindler, K. D. \& Nienhaus, G. U. (2004). Proc. Natl Acad. Sci. USA, 101, 15905-15910.

Wiedenmann, J., Oswald, F. \& Nienhaus, G. U. (2009). IUBMB Life, 61, 1029-1042.

Wilmann, P. G., Turcic, K., Battad, J. M., Wilce, M. C., Devenish, R. J., Prescott, M. \& Rossjohn, J. (2006). J. Mol. Biol. 364, 213-224. 